Artículo de investigación

ISSN: 0123-5095

E-ISSN: 2389-9441

Vol. $3-\mathrm{N}^{\mathrm{o}} 21$

Junio - diciembre, año 2017

pp. $135-164$

\title{
Educación y arte. Acerca de John Dewey
}

\author{
Education and Art. Regarding John Dewey
}

\author{
Fabio Campeotto* \\ (CONICET) Universidad de la Rioja - Argentina \\ Claudio Marcelo Viale $* * 1$ \\ (CONICET) Universidad Católica - Argentina
}

\footnotetext{
${ }^{*}$ CONICET. UNLaR. Argentina. Fabio Campeotto es licenciado en Historia y Conservación de Bienes Culturales e Historia del Arte por la Universidad de Padua. Actualmente, es becario doctoral del CONICET (Consejo Nacional de Investigaciones Científicas y Técnicas) en la Universidad Nacional de La Rioja. Argentina. Email: fcgumbo@gmail.com. Áreas de interés: estética e historia del arte. Referencias bibliográficas:

Campeotto, F. (2017). El 'baptisterio paleocristiano' de Treviso: reinterpretación de las funciones de un edificio tardo antiguo. En Anuario del Centro de Estudios Históricos "Carlos C. A. Segreti". En prensa. Marzo 2017.Dirección postal: Fabio Campeotto. Islas de los Estados 282, 5300. La Rioja. Argentina.

${ }^{* *}$ CONICET. CIJS. UNC. UNLaR. Argentina. Claudio Marcelo Viale es doctor en Filosofía por la Facultad de Filosofía y Humanidades de la Universidad Nacional de Córdoba (Argentina). Actualmente, es investigador del CONICET en la Universidad Católica de Córdoba (UAAREA CS. SOC. Y HUM. UCC 24503) en Argentina..Email: cmviale@gmail.com. Referencias bibliográficas: Viale, Claudio M. (2015). Sola fides at the Core of Varieties: Luther as Religious Genius in William James's Thought. The Pluralist. vol. 10, n. ${ }^{\circ}$ (Spring 2015), pp. 80-106. Official Journal of the Society for the Advancement of American Philosophy. University of Illinois Press.

----- (2015). Voluntarismo y self-surrender en la concepción de religión de William James. Areté. Revista Peruana de Filosofia. vol. XxvII, n. ${ }^{\circ}$ 2, 2015 pp. 209-223. Pontificia Universidad Católica de Lima.

Dirección postal: Claudio Marcelo Viale. Universidad Católica de Córdoba. Obispo Trejo 323, Centro, X5000HG, Córdoba, Argentina.
}

Educación y arte. Acerca de John Dewey,

Fabio Campeotto / Claudio Marcelo Viale - p. 135-164 


\title{
Resumen
}

Los propósitos de este artículo son dos: en primer lugar, presentar los rasgos característicos tanto de la filosofía de la educación como de la estética de Dewey y su íntima vinculación; en segundo lugar, señalar la relevancia que la influencia de Albert C. Barnes tiene para el desarrollo de las concepciones de arte y de educación del autor de Art as Experience. Para mostrar la influencia de Barnes en Dewey, tópico pasado por alto por la literatura secundaria o solo tratado superficialmente, utilizamos diversos elementos, especialmente la reseña que Dewey escribe sobre el libro de Barnes, The Art in Painting (1925), titulada "Art in Education - Education in Art" (1926) y que traducimos en este número de la revista.

Palabras clave: John Dewey; Barnes; estética; educación; arte; pragmatismo; clásico.

\begin{abstract}
This article has two objectives. Firstly, it aims to introduce the characteristics of both the philosophy of education and Dewey's aesthetics as well as their close relationship. Secondly, to point out the relevance of Albert C. Barnes's influence to develop art and education concepts over the author of Art as Experience. To demonstrate Barnes's influence over Dewey, a subject that has been overlooked or superficially analyzed in secondary literature, we use diverse elements, especially Dewey's review of Barnes's book, The Art in Painting (1925), entitled "Art in Education - Education in Art" (1926), translated in this magazine issue.
\end{abstract}

Key words: John Dewey, Albert C. Barnes, aesthetics, education, art, classical pragmatism. 


\section{INTRODUCCIÓN}

Indudablemente, uno de los principales legados de los trabajos del influyente pensador norteamericano Richard Rorty (1931-2007) fue la explícita reivindicación de la filosofía de John Dewey (1859-1952) que llevó a cabo. Su celebrado Consequences of Pragmatism (1982), como se ha reconocido en la discusión filosófica contemporánea, reavivó el interés en la obra de Dewey quien, como es bien sabido, no solo fue un filósofo de renombre hasta su deceso en 1952, sino también uno de los intelectuales públicos más ilustres en Estados Unidos en la primera mitad del siglo xx. La obra del autor de Democracy and Education, como bien presentan diversas historias de la filosofía en Norteamérica ${ }^{2}$, después de su muerte cae en un pronunciado olvido $\mathrm{y}$, aunque algunos intérpretes resaltaban la relevancia tanto del pragmatismo clásico, en general, como de los escritos de Dewey, en particular, ambas ocupaban un innegable lugar periférico en el escenario filosófico.

Ahora bien, ¿por qué fue tan influyente la interpretación que hizo Rorty de Dewey y cuál era el aspecto central que rescataba? Aunque establecer cadenas causales precisas para responder a estas preguntas es extremadamente difícil, creemos que la respuesta puede girar alrededor de dos ejes: por un lado, para Rorty recurrir a Dewey funcionaba como un antídoto ante la lejanía artificial frente a la vida práctica que aquejaba a la filosofía analítica predominante; $y$, por el otro, recuperar su figura era una apuesta del neopragmatista para ampliar el público al que se dirigía, más allá de los filósofos profesionales.

Estos dos ejes aparecen en libros posteriores al de Rorty, que nos ayudan a ilustrar lo que sostenemos. El primer ejemplo lo encontramos en Richard Bernstein, quien en su impecable The Abuse of Evil (2002) rastrea cómo se desenvuelven históricamente el falibilismo y el fundamentalismo como dos principios rectores y contrapuestos de la mentalidad norteamericana, mostrando las peligrosas raíces del belicismo de George W. Bush ${ }^{3}$. El pragmatismo clásico, en general, y el de Dewey, en particular, conforman en la estupenda pluma de Bernstein el núcleo que da vida al mejor basamento que ofrecen las tradiciones intelectuales norteamericanas para la democracia: el falibilismo. The Abuse of Evil va más allá — en la forma y en el fondo- de los confines de la filosofía académica y plantea temas de indudable interés público. El segundo ejemplo, en tanto, lo encontramos en un libro imprescindible de Martha Craven Nussbaum, Not for profit. Why Democracy

\footnotetext{
2 Véanse: Hookway (2013) y Misak (2013).

3 En relación al libro de Bernstein, véase Viale (2013).

Educación y arte. Acerca de John Dewey,

Fabio Campeotto / Claudio Marcelo Viale - p. 135-164
} 
Needs the Humanities (2010), en el que lúcidamente alerta sobre el peligro que enfrentamos en las sociedades contemporáneas a nivel global, debido a la creciente pérdida de espacio de las artes y humanidades en los currículos escolares. Al igual que el texto de Bernstein, el de Nussbaum nos ayuda a tratar - con rigor argumentativo pero escrito en un lenguaje claro y ameno - un tema esencial: el futuro de la educación y el de la democracia - inevitablemente ligados entre sí-.

La pregunta que surge, entonces, es la siguiente: ¿qué tienen en común las obras de Rorty, Bernstein y Nussbaum? A pesar de las diferencias de matices, objetivos y temáticas, todas ellas se inspiran explícitamente en la filosofía de John Dewey, con quien sienten que comparten una viva tradición común. En otros términos, tanto Rorty como Bernstein y Nussbaum buscan en la filosofía de John Dewey recursos que ayuden a dar respuesta a numerosos problemas contemporáneos. Todos ellos, en consecuencia, en mayor o menor medida trazan un vínculo entre arte, educación y democracia, tal como hizo el gran pragmatista clásico.

En ese marco, los propósitos de nuestro artículo son dos: en primer lugar, mostrar las características centrales de la filosofía de la educación y de la estética de Dewey, así como sus innegables vínculos; en segundo lugar, señalar que la estética de Dewey fue fuertemente influida por Albert C. Barnes.. Al respecto, es preciso notar que en 1926 Dewey escribe una reseña del libro de Barnes The Art in Painting, titulada "Art in Education - Education in Art", que traducimos en este número de la revista. Si bien las obras principales de Dewey cuentan con traducciones a nuestra lengua, existen innumerables textos menores (reseñas, pequeños artículos) sin traducción castellana, en los que el gran filósofo norteamericano clarifica muchas de sus concepciones teóricas. En este sentido, creemos que esta traducción que realizamos es útil ya que nos muestra claramente la relación que existe entre educación y arte para Dewey y, asimismo, nos permite entender su vínculo con Barnes, asunto central para el desarrollo de su estética y concepción de arte que, como ya mencionamos, es un vínculo que no ha sido examinado con la profundidad necesaria en la literatura secundaria.

Para llevar a cabo estos propósitos, dividimos este artículo en cuatro partes: en la primera (¿Para qué educar? Dewey y nuestro presente) realizamos una breve introducción a su filosofía de la educación; en la segunda (¿Lujo o necesidad? Dewey y la pregunta por el valor de la experiencia estética y del arte) presentamos los lineamientos generales de su estética; en la tercera par- 
te (Educación, arte, política y ¿amistad? Dewey y Albert Barnes) describimos la relación que existió entre ambos, temática que, hasta donde sabemos, es completamente inexplorada en lengua castellana; finalmente, en la última parte ("La educación en el arte - el arte en la educación". Dewey lector de Barnes) analizamos los principales núcleos conceptuales del texto traducido y presentamos nuestra conclusión: "La educación en el arte - el arte en la educación" hoy.

\section{¿PARa Qué educar? Dewey y nuestro Presente}

La filosofía de Dewey trata diversos temas desarrollados a lo largo de su extensa vida. Como es bien sabido, la educación y la pedagogía constituyen un aspecto crucial presente en ella desde sus inicios hasta sus últimos textos (véanse: Hansen 2006 y Granger 2006). En relación a esta temática, Democracy and Education es, sin lugar a dudas, un libro de referencia obligada. Publicado en 1916, mantiene una notable vigencia y es aún de extrema utilidad como herramienta teórica frente a los complejos desafíos que enfrentan en la actualidad los sistemas educativos en todo el mundo. Uno de los modos más fructíferos de entender el libro, a nuestro juicio, es examinarlo en vinculación con sus más notables reformulaciones contemporáneas. Una de ellas es, innegablemente, la que lleva a cabo Nussbaum en Not for profit. Why democracy needs the humanities (2010), libro al que hicimos referencia.

En el desarrollo de su argumentación, la intelectual de Chicago se hace la pregunta esencial que da origen (o con la cual inevitablemente se termina topando) cualquier filosofía de la educación: ¿para qué educar? - junto a esta pregunta, es casi superfluo decirlo, aparecen otras más específicas: ¿a quién educar?, ¿cómo educar?, ¿qué enseñar? - . Esta pregunta surge, entre otras cosas, porque la educación es, como explícitamente lo plantea Dewey (1998 [1916]), una necesidad:

La continuidad de toda experiencia, mediante la renovación del grupo social, es un hecho literal. La educación, en su sentido más amplio, es el medio de esta continuidad de la vida. Cada uno de los elementos constitutivos de un grupo social, tanto en una ciudad moderna como en una tribu salvaje, nace inmaduro, indefenso, sin lenguaje, creencias, ideas ni normas sociales. Cada individuo, cada unidad de portadores de la experiencia vital de su grupo desaparece con el tiempo. Y sin embargo, la vida del grupo continúa. Los hechos primarios ineluctables del nacimiento y la muerte de cada uno de los miembros constitutivos de un grupo social determinan la necesidad de la educación (p. 14, cursiva nuestra). 
En consecuencia, en todas las sociedades de hecho se educa, porque es una necesidad de reproducción vital, como bien argumenta Dewey. Las dificultades surgen, sin embargo, cuando se va más allá de lo empírico y nos adentramos en un nivel normativo. Los interrogantes que tenemos que responder, en consecuencia, son dos: ¿para qué sirve la educación?, pregunta que puede limitarse solo a un nivel empírico; ¿para qué debería servir la educación?, pregunta que va más allá de lo que efectivamente sucede y puede incluso interpelar críticamente la realidad. Al retomar explícitamente temas y argumentos propios de la concepción de educación de Dewey, Nussbaum (2010) se refiere a este ámbito normativo y sostiene que la educación debe ser útil para que los individuos logren desarrollar aptitudes necesarias para actuar eficazmente en tres ámbitos: en primer lugar, en el ámbito del trabajo, esto es, lo que ella llama "educación para la renta" (pp. 34-47); en segundo lugar, en el ámbito de la formación política de los ciudadanos, a la que denomina "educación para la democracia" (pp. 47-49); y, finalmente, para brindar herramientas que permitan responder o ayudar a pensar acerca de cómo dotamos de sentido a nuestra vida. El argumento central del estupendo libro de Nussbaum es que, en la actualidad, existe una preocupante estrechez en la definición de educación, con una fuerte tendencia a equipararla con la educación para la renta o para el trabajo, en flagrante desmedro de los otros dos ámbitos (ciudadanía y sentido) que menciona. Aunque esa tendencia creciente evidentemente varía de acuerdo al país de que se trate, o incluso de la zona de un país, esta acarrea dos peligros concretos: o bien pone en riesgo a la democracia en la que esta existe, o bien no posibilita su surgimiento en la que no existe. En 1926, Dewey (1984 [1926a]) ya había realizado un planteamiento similar al de Nussbaum en lo que concierne a la situación de la educación:

La preocupación moderna con la ciencia y la industria basada en la ciencia ha sido desastrosa; nuestra educación ha seguido el modelo que ellas han establecido. Se ha interesado en el análisis intelectual y la información formularizada, y con el entrenamiento técnico para este o aquel campo de actividad profesional, una afirmación cierta, en general, tanto del académico de las disciplinas clásicas o de literatura, de las bellas artes mismas, como de expertos en otras áreas (...). El resultado es desastroso porque refuerza la tendencia hacia la profesionalización, o al establecimiento de la mente en surcos [grooves] (p. 112, cursiva y traducciones nuestras).

Esa visión que compartimentaliza las acciones o capacidades de los seres humanos, la "(...) mente en surcos [grooves]", de la que habla Dewey, imposibilita el desarrollo de una capacidad central que todos los individuos 
de una democracia deben, en mayor o menor medida, tener: la imaginación narrativa. En los inequívocos términos de Nussbaum (2010):

El conocimiento fáctico y la lógica no alcanzan para que los ciudadanos se relacionen bien con el mundo que los rodea. La tercera capacidad del ciudadano del mundo, estrechamente vinculada con las primeras dos, es aquella que denominamos "imaginación narrativa", es decir, la capacidad de pensar cómo sería estar en el lugar de otra persona, de interpretar con inteligencia el relato de esa persona y de entender los sentimientos, los deseos y las expectativas que podría tener esa persona (p. 131-132).

Lo que tanto Dewey como Nussbaum sostienen, no es dejar de lado y/o descartar lo que esta última llama "el conocimiento fáctico y la lógica" (2010:131), y que el pragmatista clásico denomina "el análisis intelectual y la información formularizada" (Dewey, 1984 [1926a]:112). Lo que adecuadamente señalan es su radical insuficiencia. En otros términos, la democracia imperiosamente necesita que los ciudadanos desinstrumentalicen a los otros seres humanos, esto es, al menos, que traten de comprenderlos en su vasta complejidad. Nussbaum (2010) es particularmente clara al respecto:

Concebir a los otros seres humanos como entidades amplias y profundas, con pensamientos, anhelos espirituales y sentimientos propios no es un proceso automático. Por el contrario, lo más fácil es ver al otro como apenas un cuerpo, que por ende puede ser usado para nuestros propios fines, sean éstos buenos o malos. Ver un alma en ese cuerpo es un logro, un logro que encuentra apoyo en las artes y la poesía, en tanto éstas nos instan a preguntarnos por el mundo interior de esa forma que vemos y, al mismo tiempo, por nuestra propia persona y nuestro interior (pp. 131-132).

No es difícil inferir que Democracy and Education resuena en esas iluminadoras palabras. El logro sobre el que escribe Nussbaum solo se puede realizar a través de un proceso educativo adecuado y eficazmente planeado con ese fin, como bien expresó Dewey (1998 [1916]) hace poco más de una centuria:

Con el desarrollo de la civilización aumenta la distancia entre las capacidades originales de los seres inmaduros y las normas y costumbres de las personas mayores. El mero crecimiento físico, el mero dominio de las puras necesidades de subsistencia, no bastarán para reproducir la vida del grupo. Se requieren esfuerzos deliberados y trabajos reflexivos. Los seres recién nacidos no sólo desconocen, sino que son completamente indiferentes respecto a los fines y hábitos del grupo. La educación como necesidad de la vida social, que ha de hacérselos conocer e inspirarles interés activo hacia ellos. La educación, y sólo la educación, llena este vacio (pp. 14-15, cursiva nuestra). 
En la apreciación de Dewey, "los esfuerzos deliberados y los trabajos reflexivos" (Dewey, 1998 [1916]:14) que implican la existencia de un sistema educativo institucionalizado estaban totalmente descarriados en su época. Algo fundamental faltaba: "El arte y la apreciación estética es lo que falta, entendiendo por "arte" cualquier actividad selectiva por la cual las cosas concretas están organizadas de tal modo que llamen la atención hacia los valores distintivos que pueden realizar" (Dewey, 1984 [1926a]:112). En consecuencia, para el gran pragmatista, el "desastre" de la educación de su tiempo era producto de la ciega profesionalización, del mero "entrenamiento técnico para este o aquel campo de actividad profesional" (p. 113). La solución a esos problemas estaba, para Dewey, precisamente en eso esencial que faltaba: el arte y la apreciación estética. Su diagnóstico y su solución, indudablemente, no pueden haber envejecido menos.

\section{¿LuJo O NeCESIDAd? DeWey Y LA PREgunta POR EL VALOR De LA EXPERIENCIA ESTÉTICA Y DEL ARTE}

Art as Experience (1934) es a la estética de Dewey lo que Democracy and Education es a su pedagogía y su filosofía de la educación, esto es, su texto clave. De los numerosos tópicos que aparecen en el libro, queremos resaltar cuatro que son relevantes para nuestros propósitos y que están íntimamente ligados entre sí: 1) Las razones por las que Dewey concibe a la experiencia estética como primordial para su filosofía. 2) Los argumentos que esgrime al sostener que la experiencia estética y el arte para él no son un lujo sino una necesidad. 3) Su explícita crítica a la concepción del arte como objeto de museo. 4) El vínculo que establece entre arte, experiencia estética y educación, finalmente.

En relación al primero de los tópicos mencionados, en Art as Experience Dewey (2008 [1934]) escribe lo siguiente: "[la experiencia estética] (...) es la experiencia libre de las fuerzas que impiden y confunden su desarrollo como experiencia; es decir, libre de factores que subordinan la experiencia tal como se tiene, a algo que está más allá de ella misma. Entonces el filósofo debe ir a la experiencia estética para entender lo que es la experiencia" (p. 309 , cursiva nuestra).

Este es un punto clave de su pensamiento, que además se vincula con los tres tópicos del libro que tratamos. Cuando Dewey conmina al filósofo a tomar en serio la experiencia estética, apunta a que pueda llegar a percibir un rasgo característico que ella expresa mejor que nadie: la cualidad consumatoria (Dewey 2008 [1934]: caps. 7 y 8). Aunque un tratamiento exhaustivo de este 
tópico excede los límites de este trabajo, cabe destacar algunos de sus aspectos, ya que la experiencia estética constituye la piedra fundamental de su concepción filosófica. El primero a considerar es que la naturaleza de la experiencia estética debe ser buscada en un proceso, más bien en la consumación de un proceso, y por eso no puede reducirse a la mera contemplación de un producto considerado bello. Un segundo aspecto, en tanto, es que esta es una cualidad y por ello no existe una preeminencia de los productos considerados bellos en ella, sino que, como cualidad, puede estar presente en distintos tipos de experiencia. Intentaremos aclarar estos aspectos a lo largo de esta sección.

En relación al segundo tópico mencionado, y con clara vinculación con el primero, en Art as Experience Dewey (2008 [1934]) señala cómo lo estético se origina en la experiencia. En sus propias palabras:

He tratado de mostrar, en estos capítulos, que lo estético no es una intrusión ajena a la experiencia, ya sea por medio de un lujo vano o una idealidad trascendente, sino que es el desarrollo intenso y clarificado de los rasgos que pertenecen a toda experiencia completa y normal. Considero que este hecho es la única base segura para construir la teoría estética. Quedan por sugerir algunas de las implicaciones de este hecho fundamental (pp. 53-54, cursiva nuestra).

Destaquemos de este párrafo que la dimensión estética de la experiencia no es ni un "lujo vano" ni una "idealidad trascendente". ¿Cuáles son los alcances de esta afirmación? Un lujo vano se refiere a algo que no es estrictamente necesario para vivir. Para Dewey, en cambio, la experiencia estética es una necesidad vital. La idealidad trascendente, en tanto, se refiere a algo que inevitablemente está fuera de la experiencia, usualmente concebida en este formato: la experiencia ordinaria es pedestre, baja, etcétera; en cambio, la experiencia estética y el arte son sublimes. Para el autor de Experience and Nature, entonces, tanto la denominación de lujo vano como la de idealidad trascendente, o bien no captan la naturaleza de la experiencia estética, o bien la distorsionan.

El tercer tópico que mencionamos es la crítica que Dewey hace de la concepción de arte como objeto de museo ${ }^{4}$. A nuestro juicio, su argumento crítico podría ser el siguiente: ya que la experiencia estética está fuera de la

\footnotetext{
4 Este tema ha sido discutido en la literatura secundaria. Alexander (1987), por ejemplo, sostiene que "El origen del arte no puede ser encontrado en el deseo de ser alojado en un museo. En cambio, el arte se origina cuando la vida deviene plena de momentos de vitalidad inteligentemente elevada.
} 
experiencia ordinaria - en las concepciones que él critica - no es sorprendente que se encierren en un lugar particular. Sin embargo, la raíz del problema se origina, como bien piensa Dewey (2008 [1934]), en la separación radical entre experiencia estética y experiencia ordinaria: "Muchas personas protestan contra la concepción del arte como objeto de museo, sin embargo, comparten la falacia de la que brota aquella concepción. La noción popular proviene de una separación del arte de los objetos y escenas de la experiencia ordinaria, que muchos teóricos se jactan de sostener y elaborar (p. 6).

Entonces, según el pragmatista clásico, para entender la experiencia estética y el arte necesitamos un "detour", esto es, dejar a un lado los objetos o productos de arte (qua objetos y productos de arte) e intentar remitirnos a la experiencia ordinaria de la que son resultado.

Si bien, como dijimos, va más allá de las posibilidades de este artículo analizar los tres tópicos mencionados en detalle, ellos nos muestran un rasgo clave para nuestros propósitos: la experiencia estética y el arte no deberían estar en un pedestal inaccesible y no son un lujo, sino una necesidad. No solo eso, y con esto entramos en el último tópico mencionado, también Dewey plantea que esa necesidad debe vincularse con la educación. En su reseña de The Art in Painting de Barnes, por ejemplo, sostiene inequívocamente esa concepción y destaca algunos rasgos sobresalientes del libro:

Afirman que la apreciación estética inspirada y dirigida por el arte es una exigencia legítima e imperiosamente urgente del hombre común; afirman que el método, y la inteligencia, pueden ser empleados no sólo por algunos críticos, para el goce o el conocimiento de un pequeño círculo, sino para que todos puedan ser educados para obtener lo que la pintura tiene que dar. Afirman este último aspecto brindando, en general y detalladamente, un método, y mostrándolo en su funcionamiento. Por lo tanto, plantean un problema de inmensa importancia en la educación, un problema íntima y vitalmente relacionado con la mayor debilidad de la educación actual, una debilidad que afecta en manera desastrosa todas las fases de la vida contemporánea. Este es el hecho que otorga al libro una cualidad que no guarda relación con la de

Cuando la potencialidad de la experiencia se utiliza intencionalmente hacia un fin tan completo (...). Esto es lo que Dewey llama 'una experiencia'. En una experiencia, realmente llegamos a habitar el mundo; residimos en el mundo y nos apropiamos de sus significados" (p. xix, traducción nuestra). Un análisis detallado de la concepción de Dewey acerca de los museos puede verse en Constantino (2004). 
otros "tratados" sobre pintura y arte y que, en consecuencia, exige una crítica que está fuera de lo habitual (Dewey, 1984 [1926a]:115, traducción nuestra, cursiva nuestra).

Hasta ahora nos hemos enfocado en la pedagogía y en la estética del autor de Logic. A Theory of Inquiry. En la primera parte vimos por qué y en qué sentido la educación, para Dewey, es una necesidad. En la segunda parte, en tanto, examinamos por qué la experiencia estética y el arte también son, para el pragmatista clásico, una necesidad. No son, sin embargo, dos necesidades aisladas. Para retomar el título de la reseña que Dewey hace del libro de Barnes, se debe concebir el arte en la educación y la educación en el arte.

\section{Educación, ARTe, política y ¿AMistad? Dewey y Albert Barnes}

La influencia de Albert Coombs Barnes en el desarrollo de la estética deweyana ha sido a menudo ignorada, o por lo menos subestimada, por la mayoría de los especialistas en la obra de Dewey ${ }^{5}$. A pesar de las diferencias de personalidad y de carácter — Barnes era un self-made man iracundo y explosivo, rechazado por buena parte del establishment cultural; Dewey era

\footnotetext{
5 Sidney Hook (1952) escribe acerca de la amistad entre Dewey y Barnes lo siguiente: "La bondad de Dewey era tan genuina, constante, y permanente, incluso bajo la provocación, que a veces lo encontré algo opresivo. Fue casi con alivio que descubrí una grave deficiencia en él. Esa fue su indulgente amistad para Albert C. Barnes" (p. 249, traducción nuestra). Alan Ryan (1995) usa términos muy parecidos a los de Hook: "Dewey tenía un gusto por la compañía de excéntricos de todo tipo, su aparente ingenuidad, que los amigos lamentaban, puede haber sido un verdadero error de juicio más bien que una política de dar a posibles charlatanes el beneficio de la duda" (p. 207, traducción nuestra). Entre los principales estudiosos de la estética de Dewey, Abraham Kaplan (1987) ignora totalmente la influencia de Barnes en su detallada introducción de Art as Experience. Thomas M. Alexander (1987), en tanto, dedica solo un párrafo de su extensa monografía a la amistad entre los dos hombres y señala, otra vez, el carácter extraño de la relación: "En 1917 (...) el irascible millonario-empresario Albert C. Barnes, dueño de la mayor colección de Impresionistas franceses en el hemisferio occidental, desarrolló una pasión por la filosofía de Dewey. Después de leer Democracy and Education, colgó obras de Monet en su fábrica y viajó a Nueva York para asistir a las clases de Dewey. Así empezó una de las amistades más extrañas y menos predecibles en la vida de Dewey" (p. 55 , traducción nuestra). Richard Shusterman, en tanto, si por un lado reconoce la innegable influencia de las ideas de Barnes en el pensamiento estético de Dewey, por el otro considera la concepción estética de Barnes como secundaria con respeto a la de Alain Locke. Barnes, según Shusterman (2000), jugó el simple papel de "mediador" entre Locke y Dewey. En la introducción a la segunda edición de Pragmatist Aesthetics sostiene: "Casi diez años antes de Art as Experience de Dewey, [sus] temas cruciales tuvieron una formulación convincente con Alain Locke (...). A pesar de que Dewey nunca mencionó a Locke, debía haber conocido su antología histórica The New Negro. Pero también podía haber absorbido las ideas de Locke indirectamente a través del crítico de arte Albert C. Barnes, que colaboró con Locke en The New Negro y que Dewey consideraba la influencia principal de su propia estética" (p. x, traducción nuestra). Véase también Shusterman 2002:123.
}

Educación y arte. Acerca de John Dewey,

Fabio Campeotto / Claudio Marcelo Viale - p. 135-164 
un académico de temperamento humilde cuya estatura intelectual era mundialmente reconocida-, los dos tenían muchas más cosas en común de lo que generalmente se reconoce. Según George E. Hein (2011), no se puede dudar en absoluto de la amistad profunda y de la afinidad intelectual que unía a los dos hombres:

La amistad entre Dewey y Barnes, documentada por cientos de cartas que se intercambiaron, consistía no solo en frecuentes interacciones sociales (que a menudo incluían a las esposas y a los hijos de Dewey) sino también en actividades políticas comunes y un considerable intercambio intelectual. Sus discusiones sobre la teoría y la práctica estética no han sido suficientemente reconocidas por parte de quien escribió sobre Dewey o sobre Barnes. Si su relación intelectual es discutida en absoluto, los comentarios usuales son que Barnes fue influenciado por las ideas sobre la educación de Dewey, algo que Barnes reconoció a menudo. Pero la influencia opuesta de Barnes sobre la estética de Dewey no ha recibido la atención que se merece (pp. 123-124, traducción nuestra).

La relación intelectual entre el filósofo y el farmacéutico-conoisseur no fue simplemente la de un docente con su alumno: si por un lado Barnes se acercó a Dewey atraído por sus ideas políticas y pedagógicas, por el otro, supo ejercitar una influencia duradera y constante en el desarrollo del pensamiento estético de Dewey. Nuestro propósito, en esta parte del artículo, en consecuencia, es el de mostrar cómo la influencia de Barnes ha sido decisiva para Dewey no solo en la formación de una teoría estética, sino también, a través de la experiencia de la Barnes Foundation, para el establecimiento de una innegable vinculación entre arte y educación.

El intercambio intelectual entre los dos hombres, en tanto, se enfocaba principalmente en tres cuestiones, tres tópicos de importancia fundamental, ya que se entrelazan constantemente en la fase madura del pensamiento deweyano y constituyen, a su vez, los ejes centrales del pensamiento del empresario de Philadelphia. Estos son, es casi redundante decirlo, la democracia, en primer lugar; el arte y la estética, en segundo; y, finalmente, la educación (que trataremos en la cuarta parte del artículo). En términos de Hein (2017):

En Albert Barnes Dewey encontró no sólo una persona que dedicaba su vida a la educación, que en repetidas ocasiones había declarado explícitamente que su actividad educativa se basaba en la obra de Dewey, sino también un individuo que era su equivalente intelectual, un hombre que había leído una enorme cantidad, podía exponer sus opiniones con claridad y amaba discutir sobre 
ellas. Barnes y Dewey también compartían un compromiso con la política y la acción social en apoyo de causas progresistas, incluidos los derechos civiles (p. 54, traducción nuestra).

En relación a este último tema (la defensa de la democracia y de los derechos civiles) las posiciones de Dewey son bien conocidas, particularmente su idea de "democracia como una forma de vida". En Democracy and Education, el texto que acercó definitivamente Barnes al pragmatismo deweyano ${ }^{6}$, el filósofo estadounidense sostiene:

Una democracia es más que una forma de gobierno; es primariamente un modo de vivir asociado, de experiencia comunicada juntamente. La extensión en el espacio del número de individuos que participan en un interés, de modo que cada uno ha de referir su propia acción a la de los demás y considerar la acción de los demás para dar pauta y dirección a la propia, equivale a la supresión de aquellas barreras de clase, raza y territorio nacional que impiden que el hombre perciba la plena significación de su actividad (Dewey, 1998 [1916]:82).

Entre las causas sociales en las que Barnes se involucró activamente, se encuentran los movimientos "The New Negro", "The National Urban League" y la revista Opportunity, todos activos en la defensa de los derechos civiles de los afroamericanos ${ }^{7}$.

En relación al segundo tópico (el arte y la estética), sostuvimos que muy pocos estudios exponen con claridad la enorme influencia de Barnes sobre Dewey. Al comienzo de su amistad en el año 1917, Barnes ya había almacenado una fortuna considerable gracias al patentamiento de un medicamento, el argyrol. Su amor por el arte y su "obsesión por el coleccionismo" (Dolkart, 2012:10) habían surgido con anterioridad, alrededor de 1912. Es a partir de esa fecha que el empresario farmacéutico empieza a adquirir obras, que posteriormente formarán una de las colecciones artísticas más grande del mundo:

Comprando trabajos de artistas de las vanguardias europeas y estadounidenses - Paul Cézanne, Charles Demuth, Paul Gauguin, William Glackens, Vincent van Gogh, Henri Matisse, Amedeo Modigliani, Pablo Picasso, Maurice Prendergast, Pierre-Auguste Renoir, y Chaim Soutine, entre otros-, Barnes

\footnotetext{
6 "Barnes quedó particularmente impresionado por Democracy and Education poco después de que se publicó en 1916, entonces, se inscribió para asistir al seminario quincenal de Dewey 'Escrito morales, políticos y lógicos de John Stuart Mill' (...) en el semestre de otoño de 1917 (...). Antes de que terminara el semestre, Barnes había invitado a Dewey y a su esposa a visitarlo una noche y a mostrarles su colección" (Hein, 2017:59, traducción nuestra).

7 Véase Greenfield 1987:138-139.

Educación y arte. Acerca de John Dewey,

Fabio Campeotto / Claudio Marcelo Viale - p. 135-164 
demostró su audacia como coleccionista (...). El conjunto cambió a medida de que Barnes hacía nuevas adquisiciones y negocios y desarrollaba nuevos vínculos estéticos entre las colecciones, que diversificó significativamente en el curso de las décadas, con la adquisición de esculturas africanas; antigüedades; arte asiático; cerámicas de los nativos americanos; joyas y productos textiles; manuscritos; y pinturas de antiguos maestros, así como productos de las artes decorativas e industriales tanto europeas como americanas (Dolkart, 2012:9, traducción nuestra).

En 1917 Barnes ya se había convertido en uno de los coleccionistas más audaces de Estados Unidos y en uno de los expertos de arte más refinados, mientras que Dewey estaba aún muy lejos de desarrollar su propia teoría estética. Según Lawrence Dennis (1972), en efecto, "parece haber un testimonio amplio de que Dewey, ciertamente en el momento que conoció a Barnes, tenía poca o ninguna susceptibilidad a las obras de arte" (p. 327, traducción nuestra $)^{8}$. La afirmación de Dennis puede ser corroborada al recurrir a diversas fuentes. La primera de ellas es la vasta correspondencia entre los dos amigos. En una carta que Barnes envía a Dewey en 1919, por ejemplo, el empresario le propone al filósofo que dicte un seminario de estética y le ofrece su colaboración para el proyecto:

Tengo una sugerencia para tu línea académica, que creo, es práctica y muy necesaria; algo como lo siguiente: tener un seminario en la Columbia sobre la vida misma y sus fases estéticas. Todo el material que necesitas está en Democracy \& Education, Reason in Art de Santayana; sería tomar William James, McDougall y la Inteligencia Creativa en sus aspectos filosóficos y psicológicos. El arte ahora está separado de la vida, mientras que es esencialmente la vida misma (...). Ves que ahora estamos en una dialéctica socrática que abraza todo en la vida y a medida que avanzamos descubrirás que el arte y la vida son sinónimos (...). Cuando lleguemos a la "Construcción Plástica" y la "Representación Plástica" de Santayana tendremos algunos Renoir aquí para mostrar el significado - significado real, no tonterías - de los términos, dibujo, color, valores, etc. (...). Esto es sólo un bosquejo, pero verás lo que quiero decir. Arte, Ética, Política, Filosofía encajarán con la vida perfectamente. Tu seminario podría convertirse en la mejor cosa jamás intentada. Estaría encantado de cooperar cada semana para llevar a cabo el plan en forma práctica. No digas que no funcionará. Lo sé, lo he probado durante años con personas que nunca

\footnotetext{
8 Las consideraciones de Dennis se basan, en buena medida, en los testimonios del ex estudiante y colega de Dewey, Thomas Munro, y del pintor Thomas Benton, ambos de acuerdo en afirmar que Dewey no tenía ningún interés en el arte y la estética antes de conocer a Barnes. Véase Dennis 1972:327-328.
} 
fueron a la universidad sino a un taller. Por supuesto que evité ciertos términos y estaba limitado por la ausencia de lo que tú puedes ofrecer (Barnes en Hein 2017:62, traducción nuestra).

La respuesta de Dewey (2008 [1920]), que llega en 1920, no deja dudas acerca del real interés por parte del autor de Experience and Nature en el arte y la estética en aquellos años:

Me interesó tu sugerencia sobre un seminario de estética. Pero no puedo involucrarme en eso. Siempre he evitado la estética, el por qué no lo sé (...). Y ahora tengo una repulsión bastante firme en contra de toda la discusión estética. Me hace sentir precisamente como se siente el hombre de inteligencia promedio acerca de toda la discusión filosófica (traducción nuestra).

Dewey parece declinar la sugerencia de Barnes porque, a principios de los años 20 , no se sentía suficientemente preparado —o interesado- para lidiar con la estética y el arte.

La segunda fuente, en tanto, son los escritos de Barnes y Dewey. En un artículo de 1949 y nunca desmentido por Dewey, el coleccionista de arte sostiene que el interés de Dewey en las artes fue despertando luego de una discusión que tuvieron acerca de la Quinta Sinfonía de Beethoven:

Los escritos de Dewey anteriores al evento que acabamos de citar muestran muy poca atención a la filosofía del arte o a la experiencia estética per se. La música puede, o puede no haber sido el comienzo de su interés activo, pero desde luego, a partir de esa fecha, las visitas de Dewey a nuestra galería fueron más frecuentes y nuestras discusiones delante de pinturas eran siempre el rasgo dominante. Con el tiempo, sus observaciones demostraban que estaba tratando de percibir no sólo las indicaciones objetivas de la experiencia de un pintor en particular, como revelado por la forma, sino la individualidad de esa experiencia determinada en consideración de sus relaciones con la tradición de la pintura en general. Llegó a esto por asistir a las clases de verano dictadas por un integrante de nuestro personal en las principales galerías de Europa (Barnes, 1949:4, traducción nuestra).

En tanto, en los respectivos prefacios a sus libros de estética (The Art in Painting de Barnes de 1925 y Art as Experience de Dewey de 1934), ambos reconocen explícitamente la influencia del otro. En el primero, Barnes dedica su libro al amigo, "cuyas concepciones de experiencia, método y educación inspiraron el trabajo del cual este libro es parte" (Barnes, 1925, traducción nuestra). Más extensa y articulada es la dedicatoria que publica Dewey (2008 [1934]), nueve años después, para Barnes y su Fundación educativa:

Educación y arte. Acerca de John Dewey, 
Con todo, mi gran deuda es con el doctor A. C. Barnes. Ha leído cada uno de los capítulos y las referencias que hay sobre su obra en este libro tan sólo son una pequeña parte de la deuda que tengo con él. He disfrutado del beneficio de sus conversaciones durante varios años, muchas de las cuales fueron en presencia de su colección, sin rival, de pinturas. La influencia de estas conversaciones, junto con la de sus libros, ha sido el factor principal en la formación de mi propio pensamiento sobre la estética. Todo lo que hay de bueno en este volumen se debe más de lo que yo pudiera decir a la gran obra educacional llevada a cabo en la Barnes Foundation. Esta obra, en su calidad de iniciadora, es comparable a la mejor que se haya hecho en cualquier campo, durante la generación presente, sin exceptuar el de la ciencia. Me gustaría pensar que este volumen es una fase de la extensa influencia que está ejerciendo la Fundación (p. 2, cursiva nuestra).

Otro ejemplo, en tanto, lo encontramos en un artículo de Dewey de 1948, en el cual responde al incisivo ataque del filósofo italiano Benedetto Croce y vuelve a reconocer explícitamente el legado barnesiano. En un artículo publicado en la revista italiana La Critica en 1940 (traducido luego al inglés en 1948 y publicado en el Journal of Aesthetics and Art Criticism. Véase: Croce, 1948), el filósofo napolitano expresa toda su "insatisfacción por la escasez y vaguedad de referencias a la tradición" filosófica en Art as Experience (Croce, 1940:348). Una afirmación que se hace aún más grave, dado que se acerca a la acusación de plagio, cuando Croce reconoce dieciocho puntos en común entre su propia teoría estética — de matriz idealista - y la pragmatista de Dewey. El núcleo central de la respuesta del autor de $\mathrm{ACom}$ mon Faith gira alrededor de su deuda con las ideas de Barnes:

He aprendido muy poco de lo que se ha escrito en nombre de la Filosofía del Arte y de la Estética, ya que me parecía subordinar el arte a la filosofía (...). No creo exagerar en decir que le debo más a los libros sobre las artes plásticas escritos por el hombre al que dediqué mi libro, Albert C. Barnes, que a todos los tratados oficiales sobre el arte escritos por filósofos (Dewey, 1989 [1948]:100, traducción nuestra).

Más adelante, en el texto, Dewey menciona nuevamente al amigo, particularmente en respuesta a la acusación de Croce de usar su misma concepción de "que para un juicio sobre el arte es indispensable el conocimiento de la historia" (Croce, 1940: 350):

Hay otro caso en el que hay que ser preciso: el último mencionado por mi crítico, "que el conocimiento histórico es indispensable para el juicio sobre el arte". Si los libros del Dr. Barnes están disponibles en Italia y Croce se 
preocupa por consultarlos, encontrará allí una insistencia en la importancia de la continuidad de la tradición tanto en la producción de las obras de arte, así como en la apreciación crítica de ellas. De hecho, toda la teoría del juicio expresada en mi libro no es más que un eco de lo que se puede encontrar en lo que el Dr. Barnes ha dicho acerca de las artes plásticas, y que yo considero como una fuente de instrucción con respecto a todas las artes (Dewey 1989 [1948]:101, traducción nuestra. Cursiva nuestra).

La disputa entre Croce y Dewey ha sido objeto de estudios detallados, algunos más favorables al filósofo norteamericano (véase, particularmente: Alexander, 1987: cap. 1), otros más dispuestos a defender la posición del italiano (por ejemplo, Douglas, 1970). En todos los casos, los autores no tratan siquiera superficialmente lo que constituye el núcleo de la respuesta de Dewey, esto es, la enorme influencia que tuvo Barnes para su concepción estética. Como sostiene David Granger, el legado de Barnes en la estética del autor de Art as Experience es evidente, principalmente en las partes del libro en las que la discusión filosófica adquiere los rasgos de una crítica formalista. Una parte de las ideas de Barnes sobre el arte, en efecto, procede de los escritos de los formalistas ingleses Roger Fry (1866-1934) y Clive Bell (1881-1964):

Las partes de Art as Experience que desarrollan temas introducidos previamente en Experience and Nature probablemente podrían haber sido escritas sin que Dewey hubiera conocido a Barnes. Pero el comentario detallado acerca de las propiedades formales de las artes plásticas y de los diferentes medios del arte, especialmente en relación con piezas específicas de pintura y escultura, con toda probabilidad dependían del conocimiento de Barnes (...). Curiosamente, estas son algunas de las ideas formalistas (...) por las que Art as Experience es a menudo criticado como un "retroceso" al idealismo (Granger, 2007:55-56, traducción nuestra).

En las páginas en las que Dewey discute sobre los conceptos típicamente formalistas de ritmo, armonía, forma, etcétera, (principalmente en los capítulos 6 y 7), la influencia de Barnes es notoria. La vasta correspondencia entre los dos intelectuales nos ayuda nuevamente a reconstruir el origen del legado barnesiano en este punto en particular. En una carta que Dewey (2008 [1931]) envía al empresario en marzo de 1931, es decir, en medio de las famosas lecciones de Harvard que originaron Art as Experience, el filósofo pragmatista se refiere al desarrollo de las mismas en los siguientes términos: 
Gracias por tus contribuciones útiles. Hice dos conferencias con el material sobre forma-ritmo, balance (peso) y probablemente no daré ninguna charla sobre la crítica, aunque por supuesto incluiré el tema en el libro, y usaré tus sugerencias junto con las de las cartas y conversaciones futuras. Y por supuesto tu memorandum me ayudará a mantenerme en el tema principal mientras manejo otros tópicos. Mantengo tu libro a mi lado y lo uso frecuentemente (traducción nuestra, cursiva nuestra).

A nuestro juicio, la subestimación de la influencia barnesiana en el desarrollo de las teorías estéticas de Dewey puede ser adjudicada a dos factores: en primer lugar, a la falta de integración del empresario de Filadelfia en ambientes académicos, debido a su carácter iracundo y sus ínfulas despóticas, por un lado, y su obstinación anti-establishment, por el otro; en segundo lugar, a su innegable concepción vanguardista del arte, que lo llevaba fuera de esos círculos. Las palabras de Harold McWhinnie (1994), en tanto, pueden servir para rescatar, aunque sea parcialmente, su figura, frecuentemente olvidada y/o maltratada por la crítica:

Barnes no era ajeno a la estética. Sus ideas eran parte de la tradición estética (aesthetic mainstream) de principios del siglo veinte. De sus escritos aflora el retrato evidente de un hombre que poseía un intelecto claro y analítico, tenía un buen ojo por el arte de principios del siglo veinte, y tenía una idea clara del arte, del valor estético y del proceso creativo (p. 23, traducción nuestra).

\section{“LA EDUCACIÓN EN EL ARTE - EL ARTE EN LA EDUCACIÓN". DEWEY LECTOR DE BARNES}

Como sostuvimos con anterioridad, existen numerosos escritos menores de Dewey (reseñas, pequeños artículos) que no están traducidos al castellano y que son ilustrativos de las concepciones del gran pragmatista clásico. Uno de ellos es la reseña, escrita en 1926, del libro de Albert C. Barnes The Art in Painting, que traducimos en este número 21, Vol. 3 de la revista Cuestiones de Filosofía, titulada "La educación en el arte - el arte en la educación. Esta comienza refiriéndose a otra que escribe Dewey también en 1926 sobre el notable libro de Whitehead, Science and the Modern World, titulada "The Changing Intellectual Climate" (Dewey 1984 [1926b]:222-6). El texto de Whitehead señala abiertamente, y Dewey lo refrenda, el modo absurdo en el que son concebidos los valores de su época: o bien son los burdos o toscos de los individuos ordinarios, o bien son los específicos de los instruidos. En sus propios términos: 
Existe algo entre los toscos valores propios [gross specialised values] del hombre práctico y los finos valores específicos [thin specialised values] del mero académico. Ambos tipos han perdido algo y si se ubican los dos conjuntos de valores a la par, no se obtienen los elementos perdidos. Lo que se requiere es una apreciación de la infinita variedad de los vívidos valores adquiridos [achieved] por un organismo en su propio ambiente. Cuando entiendes todo acerca del sol y todo acerca de la atmósfera y todo sobre la rotación de la tierra, aun puedes perder el fulgor del atardecer. No existe un sustituto de la percepción directa de la realización concreta [concrete achievement] de una cosa en su realidad. Queremos hechos concretos con una potente luz iluminando lo que es relevante de su hermosura (Whitehead, 1948 [1925]:199).

Eso que existe entre ambos valores mencionados es lo que debería ser captado por la apreciación estética, según señalan correctamente Whitehead y Dewey. Para el autor de Art as Experience (y con esto entramos en el tercer tópico, arte y educación, que mencionamos páginas atrás y que vincula a Dewey con Barnes), si se intenta comprender las razones por las cuales los valores se conciben de un modo tan inapropiado, se llega a la conclusión de que la tendencia a la estrecha profesionalización, esto es, a concebir a la "mente en surcos [grooves]" (Dewey, 1984 [1926a]:113), juega un rol crucial. Esta forma de educación impide el surgimiento de lo que se necesita, esto es, en términos de Whitehead (1948 [1925]): “(...) una apreciación de la infinita variedad de los vívidos valores adquiridos [achieved] por un organismo en su propio ambiente" (p. 199). En este punto, Dewey conecta la reseña que escribió acerca del libro de Whitehead con la que escribe sobre el libro de Barnes: para superar la "catastrófica" situación de la educación de su época, se debe concebirla de una manera que nos permita captar esos valores $\mathrm{y}$, en ese sentido, The Art in Painting es fundamental ya que "(...) está escrito desde la convicción de que el arte tal como es mostrado en la pintura es inherentemente educativo" (Dewey 1984 [1926a]:113-114). A su vez, el otro punto fundamental a rescatar de la reseña de Dewey es su afirmación de que se necesita un método preciso para poder apreciar genuinamente el arte.

La reseña de The Art in Painting, entonces, nos muestra dos aspectos del pensamiento de Barnes que Dewey señala y luego retoma principalmente en Art as Experience: 1) El énfasis en el aspecto cognoscitivo de la experiencia estética. 2) La necesidad de un método de educación estética. Con respecto al primer punto, la concepción de Barnes (1929) está claramente expresada en el siguiente párrafo: 
El arte no es un asunto trivial, no es un artefacto para el entretenimiento de diletantes, ni una tapicería para las casas de los ricos, más bien es una fuente de conocimiento [insight] del mundo, para la cual no hay y no puede haber un sustituto, [fuente] que todas las personas que tienen el conocimiento [insight] necesario tendrían que compartir ( $\mathrm{p}$. v-vi, traducción nuestra, cursiva nuestra).

Este es un punto esencial que comparten Barnes y Dewey - y que ha sido recuperado explícitamente por Nussbaum—, a saber, la experiencia estética y el arte son una fuente de conocimiento. Ahora bien, ¿cómo se relaciona esta forma de concebir el arte con la idea de método de enseñanza que describe Dewey al seguir a Barnes? La educación era un tema crucial para ambos. George E. Hein (2011) señala claramente este aspecto del pensamiento y de la acción de Albert C. Barnes:

[Barnes] no era sólo un coleccionista (después de que se hizo enormemente rico), sino también un industrial ilustrado [enlighted] que se tomó en serio el bienestar de sus empleados y quería mejorar sus vidas. Sus esfuerzos educativos probablemente comenzaron seriamente después de convertirse en propietario único de la A. C. Barnes Company en 1908 en Filadelfia, su ciudad natal. Realizó seminarios con sus trabajadores durante el horario de trabajo que incluían William James, Dewey y Santayana, y después de que comenzó a coleccionar pinturas a principios de 1912, colgó algunas de ellas en la fábrica (p. 128, traducción nuestra).

Una sociedad educada en los principios de la democracia y en la apreciación estética era la preocupación principal del empresario de Filadelfia. Para Barnes la educación, y principalmente la educación estética, es el medio más efectivo para lograr una sensible mejora de las condiciones de vida de la sociedad entera. En una carta enviada a Alice Dewey en 1920, por ejemplo, Barnes describe claramente la visión de la educación que tuvo a lo largo de su vida:

Mi principal interés siempre ha sido la educación, primero para mí, luego para los menos afortunados alrededor de nosotros, entonces para el público en general. (...) Desde que tenía 11 años hasta ahora me he interesado en la educación - especialmente ese tipo de educación que considera a la experiencia como su mejor maestra- (Barnes en Hein, 2011:128, traducción nuestra, cursiva nuestra). 
Luego de algunos experimentos educativos en su fábrica, que involucraban directamente a sus empleados ${ }^{9}$, Barnes decidió trasladar su enorme colección a una Fundación, la famosa Barnes Foundation, que abrió sus puertas en el suburbio de Merion en 1922. Aunque hoy, en su nueva sede de Filadelfia, la Barnes Foundation se parece a un museo - para no decir que se ha convertido en un museo en casi todos los aspectos-, los propósitos originales de su fundador eran radicalmente diferentes. El objetivo principal de Barnes era el de crear una institución educativa que, al enseñar a ver el arte, apuntara a mejorar sensiblemente la vida de sus estudiantes. La concepción negativa de Barnes acerca de los museos modernos era bien conocida. Como sostiene Walter Feinberg (2013):

Barnes era escéptico sobre el valor de los museos modernos y no sólo porque los veía como palacios para la admiración frívola, y la adoración falsa (...). Era también escéptico porque los veía fomentar una concepción pasiva de ver, y porque creía que los museos tenían una influencia perniciosa en la educación artística (p. 62, traducción nuestra).

Como ha señalado Tracie Costantino, la invectiva de Dewey $-\mathrm{y}$, en consecuencia, la desconfianza de Barnes - en contra de los museos no se refiere a las instituciones culturales per se, más bien a su tendencia generalizada y antieducativa de promover una percepción pasiva de las calidades estéticas de las obras. Constantino (2004) arguye, convincentemente, acerca de las precauciones que tenemos que tener para no malinterpretar a Dewey $-\mathrm{y}$ análogamente a Barnes - en este punto:

A pesar de la crítica a los museos norteamericanos como se encontraban a principios del siglo veinte, Dewey reconoció el potencial educativo de los museos como instituciones dedicadas al mejoramiento de la sociedad, un punto que discutió varias veces en su carrera. Esta función educativa era relevante para ambos niños y adultos, y se enfocaba en llevar el arte en la vida cotidiana para entrenar la percepción estética y eliminar la distinción entre bellas artes y artes aplicadas (p. 400, traducción nuestra).

\footnotetext{
9 “[Barnes] involucró a su fuerza de trabajo en su planta química, racialmente heterogénea y con una educación mínima, en la planificación y organización de su trabajo, reduciendo así la jornada laboral de diez horas a seis horas. En el tiempo ahorrado, Barnes ofrecía a los trabajadores conferencias sobre una gran variedad de temas y autores incluyendo obras de Dewey. Curiosamente, las conferencias no fueron sobre cuestiones técnicas pensadas para mejorar la productividad, sino más bien sobre temas de psicología, filosofía y estética" (Feinberg, 2013:63, traducción nuestra).
}

Educación y arte. Acerca de John Dewey, 
Al afirmar la centralidad de la pedagogía de Dewey y nombrar al prestigioso amigo como director de la Fundación ${ }^{10}$, Barnes apuntaba a ofrecer una alternativa concreta en el campo de la educación artística: "La filosofía de la educación de John Dewey se basa en el axioma de que los elementos esenciales de una forma de vida democrática, el método científico como inteligencia trabajando, arte, educación, están todos relacionados entre sí en un todo orgánico" (Barnes, 1946:160, traducción y cursiva nuestras).

Los términos que mencionamos en este párrafo (método científico, arte y educación) constituyen los pilares sobre los que Barnes construye su famoso "método" de educación estética (Scientific aesthetic method, Barnes y De Mazia, 1935:xi), que Dewey reiteradamente menciona en su reseña. El objetivo del método es que el alumno logre obtener una visión —o percepciónde la obra de arte comparable con la del artista: "to see as the artist sees" (Barnes, 1925:7). En otras palabras, Barnes (1925) pretendía ofrecer a sus estudiantes las herramientas para una evaluación objetiva de las obras de arte. En sus propios términos:

Percibimos sólo lo que hemos aprendido a buscar, tanto en la vida como en el arte (...). La experiencia del artista surge de un fondo particular, un conjunto de intereses y hábitos de percepción, que, al igual que los hábitos de pensamiento del científico, son potencialmente compartibles por otros individuos. Sólo son compartibles, sin embargo, si uno está dispuesto a hacer el esfuerzo necesario para adquirir un conjunto de hábitos comparables. Ver como el artista ve es un logro para el que no hay atajo (pp. 6-7, traducción nuestra).

Dewey (2008 [1934]) retoma esta misma concepción de Barnes, casi una década más tarde, en el tercer capítulo de Art as Experience:

Para percibir, un contemplador debe crear su propia experiencia. Y esta creación debe incluir relaciones comparables a las que sintió el creador. No son las mismas en sentido literal. No obstante, en el contemplador, como en el artista, debe producirse un ordenamiento de los elementos del todo que es, en su forma, aunque no en los detalles, el mismo proceso de organización del creador de la obra experimentada conscientemente. Sin un acto de recreación,

\footnotetext{
${ }^{10}$ Acerca de los respectivos roles de Barnes y Dewey en el manejo de la Fundación, McWhinnie (1994) sostiene lo siguiente: "Durante toda su larga vida John Dewey fue el director de la Fundación y fue uno de los asesores artísticos de Barnes, aunque más en término de desempeño educativo que en la adquisición de obras de arte. Barnes dependía de Dewey por el soporte educacional y filosófico de la Fundación y de su trabajo, pero se reservó por sí mismo la selección de las obras de arte" (p. 33).
} 
el objeto no es percibido como obra de arte. El artista selecciona, simplifica, aclara, abrevia y condensa de acuerdo con su interés; y el contemplador debe pasar por estas operaciones, de acuerdo con su punto de vista y su interés (...). Por parte del perceptor hay un trabajo que hacer como lo hay por parte del artista. El que por pereza, vanidad o convención rígida no haga este trabajo no verá ni oirá. Su apreciación será una mezcla de jirones del saber, conforme a las normas de la admiración convencional, y una confusa, aun cuando pudiera ser genuina, excitación emocional (pp. 62-63).

La percepción, tanto para Barnes como para Dewey, no es un mero reconocimiento ${ }^{11}$. Implica necesariamente que el observador se involucre activamente en los problemas del artista:

Esto no significa que el espectador necesita conocer la vida personal del artista - la relación de Picasso con las mujeres, la relación de Van Gogh y Gauguin. Significa que el espectador debe comprender los problemas que el artista ha enfrentado como artista; las herramientas y tradiciones disponibles, las posibilidades de color, de las formas y la razón por la cual el artista las pone en relación unas con otras en la manera en que lo hizo- lo que Dewey y Barnes llaman la forma de la obra. Percibir implica entender las posibles trayectorias que el artista podría haber tomado, las limitaciones que estaban operando y las razones para hacer algunas elecciones y no otras (Finberg, 2013:66, traducción nuestra, cursiva nuestra).

Según el "método Barnes", entonces, cualquier obra de arte puede ser percibida y apreciada a través del análisis de sus formas plásticas o, en otros términos, de sus elementos formales fundamentales: el color, la línea, la luz y el espacio. Las palabras de Dewey (2008 [1934]) pueden ser leídas como evocadoras, otra vez, de la enseñanza de su amigo:

En cualquier percepción visual ordinaria, vemos por medio de la luz, distinguimos por medio de los colores reflejados y refractados, esto es evidente. No obstante, con las percepciones ordinarias, este medio que es el color está mezclado, adulterado. Mientras vemos, también oímos, sentimos presiones y calor o frío. En una pintura, el color presenta la escena sin estas mezclas e

\footnotetext{
${ }^{11}$ Acerca de la diferencia entre percepción-visión y mero reconocimiento, Dewey (2008 [1934]) afirma: "No es tan fácil en el caso del espectador, entender la unión íntima entre el hacer y el padecer, como en el caso del productor. Solemos suponer que el espectador asimila tan sólo lo que está concluido, y no advertimos que este asimilar implica actividades comparables a las del creador. Sin embargo, receptividad no es pasividad. Es también un proceso que consiste en una serie de actos de respuesta que se acumulan, hasta llegar a la satisfacción objetiva. De otra manera no es percepción, sino reconocimiento, y la diferencia entre las dos es inmensa" (p. 60).
}

Educación y arte. Acerca de John Dewey, 
impurezas. Son parte de la escoria que se elimina y abandona en un acto de expresión intensificada. El medio se hace solamente color, exaltando su expresividad y energía, puesto que sólo el color debe conducir ahora las cualidades del movimiento, tacto, sonido, etc., que están físicamente presentes por cuenta propia en la visión ordinaria (p. 221).

Otro aspecto innovador del "método Barnes" es la presentación de su colección, que escapa de todas reglas y conveniencias de la museografía tradicional. Barnes no entendía las obras de su colección como pinturas separadas, sino como unidades de pared (wall unit). Las pinturas no seguían un orden cronológico o por escuelas y nacionalidades: cada pintura tenía, más bien, vínculos formales con la precedente y la sucesiva. Violette De Mazia (18961988), principal colaboradora de Barnes y coautora de numerosas publicaciones, describe así esta particular técnica expositiva:

Debido a las características que pertenecen tanto a las pinturas y a los objetos seleccionados como a su organización, el espectador, en su percepción "informada", es fácilmente llevado a transferir cualidades que aparecen en un elemento en la composición de otros y comprender, a través del ejemplo específico presentado, el significado de unidad y variedad y el funcionamiento de las cualidades transferidas [transferred qualities] en la conquista de la unidad (De Mazia 1988:6, traducción nuestra).

Esta nueva manera de organizar las obras se ha revelado particularmente significativa a raíz de que las mismas tendencias artísticas de vanguardia se estaban alejando progresivamente de una concepción tradicional y académica del arte:

La prioridad de la presentación de la colección de Barnes era la de acabar con la superioridad presunta del arte europeo del pasado con respecto al arte de sus contemporáneos. El método de presentación era un esfuerzo de enseñar la unicidad de la formulación estética de Barnes a visitadores con o sin una formación escolar avanzada. Subrayando yuxtaposiciones sorpresivas entre productos de las bellas artes y "artesanales", así como de arte occidental y no occidental, y mostrando trabajos significativos de la época renacentista al lado de exposiciones modernistas de vanguardias, Barnes estimuló un amplio grupo de espectadores a ver similitudes en el arte de distintas culturas y épocas históricas (Wexler 2013:27, traducción nuestra). 
Un ilustre testimonio de la eficacia del "método Barnes" llega por parte de uno de los más importantes exponentes de la vanguardia europea de comienzo del siglo xx, Henri Matisse:

Una de las cosas más impresionantes de Estados Unidos es la colección de Barnes, que se exhibe en un espíritu muy favorable para la formación de los artistas norteamericanos. Allí las pinturas de los antiguos maestros están juntas con las de los modernos, un Aduanero Rousseau cerca de un Primitivo, y esta unión ayuda a los estudiantes a entender muchas cosas que las academias no enseñan. Esta colección presenta las pinturas en completa honestidad, algo que no es frecuente en Estados Unidos. La Barnes Foundation logrará destruir la presentación artificiosa y despreciable de otras colecciones, donde las imágenes son difíciles de ver expuestas hipócritamente a la luz misteriosa de un templo o de una catedral. Según la estética norteamericana corriente, esta presentación busca introducir un cierto misterio, que se supone favorable, entre el espectador y la obra, que es en realidad una gran equivocación (Matisse, 1995 [1930]:92, traducción nuestra, cursiva nuestra).

La parte del discurso del famoso pintor francés que resaltamos en cursiva podría bien pertenecer al mismo Dewey. En 1934, en efecto, el filósofo se expresa en términos muy parecidos cuando habla de las tendencias dominantes en la estética de su época:

Las ideas que colocan el arte en un pedestal remoto penetran tan sutilmente y están tan extendidas, que muchas personas sentirán repugnancia más bien que agrado, si se les dice que gozan en sus acostumbradas recreaciones, en parte al menos, por su cualidad estética (...). Porque cuando lo que [el hombre] conoce como arte se relega al museo o a la galería, el incontenible impulso hacia experiencias que se pueden gozar en sí mismas encuentra tantos escapes cuantos el ambiente provee (Dewey 2008 (1934]:6).

\section{CONCLUSIÓN: "LA EDUCACIÓN EN EL ARTE - EL ARTE EN LA EDUCACIÓN" HOY}

Como sostuvimos en las páginas iniciales de este artículo, nos proponíamos en este trabajo dos cosas: en primer lugar, presentar los rasgos centrales tanto de la filosofía de la educación como de la estética de Dewey y los vínculos que ambas tienen; en segundo lugar, mostrar la importancia de la influencia que Albert Barnes tiene sobre la filosofía de Dewey, tópico que o bien es

Educación y arte. Acerca de John Dewey, 
descuidado por la literatura secundaria, o bien es tratado superficialmente. Para llevar a cabo esos propósitos nos remitimos (en la primer parte ¿Para qué educar? Dewey y nuestro presente) a ciertos pasajes de Democracy and Education y a la interpretación contemporánea que hace de ese libro Martha Nussbaum en Not for Profit. En la segunda parte del artículo (¿Lujo o necesidad? Dewey y la pregunta por el valor de la experiencia estética y el arte), analizamos algunos de los ejes principales de Art as Experience relevantes para nuestra argumentación. Una vez que presentamos la filosofía de la educación y la estética de Dewey de manera general, en la tercera parte del artículo (Educación, arte, política y ¿amistad? Dewey y Albert Barnes) nos concentramos en un aspecto más específico y examinamos la relación entre ambos a partir de dos fuentes: su correspondencia, por un lado y las referencias mutuas que encontramos en sus textos, por el otro. En tanto, en la última parte ("La educación en el arte - el arte en la educación". Dewey lector de Barnes), analizamos dos núcleos conceptuales de la reseña que escribe Dewey y que traducimos para este dossier: que existe un vínculo necesario entre arte y educación, y, además, que es fundamental para ese vínculo la existencia de un método.

Estos dos últimos núcleos mencionados son fundamentales, debido a dos razones: en primer lugar, nos permiten comprender mejor la filosofía de Dewey a partir del reconocimiento de la influencia que recibe de Albert Barnes; en segundo lugar, nos muestra la vigencia de la obra del pragmatista clásico para la discusión contemporánea de temas relativos a la educación y el arte. La obra de Barnes, a nuestro juicio, es esencial para entender el papel cognoscitivo que el arte debe tener en la educación contemporánea. En términos de Dewey (1984 [1926c]):

La presentación de un criterio objetivo de evaluación de pinturas como el descrito por Barnes, hará posible en el tiempo un adecuado análisis psicológico, incluso fisiológico, de las respuestas estéticas de los espectadores, de modo que la apreciación de las pinturas ya no será más una cuestión de gustos privados y absolutos" (p. 109). 


\section{REFERENCIAS}

Alexander, T. M. (1987). John Dewey's Theory of Art, Experience and Nature. The Horizons of Feeling. Albany: State University of New York Press.

Barnes, A. C. (1925). The Art in Painting. Merion: Barnes Foundation Press.

----- (1949). Dewey and Art. New Leader, 32, pp. S-4.

(1929). Preface. Art and Education. J. Dewey, A.C. Barnes et. al. (Eds.). Merion: Barnes Foundation Press, pp. v-vii.

------ y De Mazia, V. (1935). The Art of Renoir. Merion: Barnes Foundation Press.

Bernstein, R. (2006). El Abuso del mal: la corrupción de la política y la religión desde el 11/9. (Trad. A. Vassallo, W. I. Weinstahl). Buenos Aires: Katz.

Costantino, T. E. (2004). Training Aesthetic Perception: John Dewey on the Educational Role of the Art Museum. Educational Theory, 54 (4), pp. 399-417.

Croce, B. (1930). Intorno all'Estetica del Dewey. La Critica, 38, pp. 348353.

----- (1948). On the Aesthetics of Dewey. The Journal of Aesthetics and Art Criticism, 6 (3), pp. 203-207.

De Mazia, V. (1988). The Barnes Foundation: The Display of Its Collection. Merion: The Barnes Foundation Press.

Dennis, L. J., (1972). Dewey's Debt to Albert Coombs Barnes. Educational Theory, 22 (3), pp. 325-333.

Dewey, J. (1984 [1926]a). Art in Education - Education in Art. J. A. Boydston (Ed.), The Collected Works of John Dewey. The Later Works of John Dewey. vol. 2 (1925-1927). (pp. 111-115). Carbondale and Edwardsvi1le: Southern Illinois University Press.

------ (1984 [1926]b). The Changing Intellectual Climate. J. A. Boydston (Ed.), The Collected Works of John Dewey. The Later Works of John

Educación y arte. Acerca de John Dewey,

Fabio Campeotto / Claudio Marcelo Viale - p. 135-164

161 
Dewey. vol. 2 (1925-1927). (pp. 222-226). Carbondale and Edwardsvi1le: Southern Illinois University Press.

------ (1984 [1926]c). Affective Thought. J. A. Boydston (Ed.), The Collected Works of John Dewey. The Later Works of John Dewey. vol. 2 (19251927). (pp. 104-110). Carbondale and Edwardsville: Southern Illinois University Press.

------ (1987 [1934]). Art as Experience. J. A. Boydston (Ed.), The Collected Works of John Dewey, The Later Works. vol. 10 (1934). Carbondale and Edwardsville: Southern Illinois University Press.

----- (1989 [1948]). A Comment on the Foregoing Criticism. J. A. Boydston (Ed.), The Collected Works of John Dewey, The Later Works of John Dewey. vol. 15 (1942-1948). (pp. 98-101). Carbondale and Edwardsville: Southern Illinois University Press.

----- (1998 [1916]). Democracia y educación. (Trad. L. Luzuriaga). Madrid: Morata.

----- (2008 [1920]). John Dewey to Albert C. Barnes. L. Hickman (ed.). The Correspondence of John Dewey. vol. 2: 1919-1939. Electronic Edition. Date: 1920.01.15. Number: 04091.

----- (2008 [1931]). John Dewey to Albert C. Barnes. L. Hickman (ed.). The Correspondence of John Dewey. vol. 2: 1919-1939. Electronic Edition. Date: 1931.03.09. Number: 04292.

----- (2008 [1934]). El arte como experiencia. (Trad. J. Claramonte). Barcelona: Paidós.

Dolkart, J. F. (2012). To See as the Artist Sees: Albert C. Barnes and the Experiment in Education. J. F. Dolkart, M. Lucy, D. Gillman (Eds.), The Barnes Foundation Masterworks (pp. 9-29). Nueva York: Skira-Rizzoli.

Douglas, G. H. (1970). A Reconsideration of Dewey-Croce exchange. Journal of Aesthetics and Art Criticism, 28 (4). pp. 497-504.

Feinberg, W. (2013). Saving a Progressive Vision: Moving the Barnes Collection. International Journal of Progressive Education, 9 (1). pp. 59-72. 
Granger, D. A. (2006). John Dewey, Robert Pirsig and the Art of Living: Revisioning Aesthetic Education. Nueva York: Palgrave MacMillan.

------ (2007). A "Scientific Aesthetic" Method: John Dewey, Albert Barnes and the Question of Aesthetic Formalism. Education and Culture, 23 (2). pp. 52-56.

Greenfield, H. (1987). The Devil and Dr. Barnes. Nueva York: Viking.

Hansen, D. T. (Ed.). (2006). John Dewey and Our Educational Prospect: a Critical Engagement with Dewey's Democracy and Education. Albany: State University of New York Press.

Hein, G. E. (2011). Dewey's Debt to Barnes. Curator: The Museum Journal, 54 (2). pp. 123-139.

------ (2017). John Dewey and Albert C. Barnes: a Deep and Mutually Rewarding Friendship. Dewey Studies, 1 (1). pp. 44-78.

Hook, S. (1952). Some Memories of John Dewey. Commentary, 14 (3). pp. 245-253.

Hookway, Ch. (2013). Pragmatism. Stanford Encyclopedia of Philosophy. Revisado el 08 de agosto de 2017. En https://plato.stanford.edu/entries/ pragmatism/.

Kaplan, A. (1987). Introduction. J. A. Boydston (Ed.). Art as Experience. The Collected Works of John Dewey, The Later Works, Vol. 10 (1934) (pp. vii-xxxiii). Carbondale and Edwardsville: Southern Illinois University Press.

Locke, A. (1968 [1925]). The New Negro. A. Locke (Ed.). The New Negro (pp. 3-16). Nueva York: Atheneum.

Matisse, H. (1993 [1930]). Statement to Tériade: On Travel, 1930. J. Flam (Ed.). Matisse on Art (pp. 87-92). Berkeley y Los Ángeles: California University Press.

McWhinnie, H. (1994). Some Reflections on the Barnes Collection. Art Education, 47 (6). pp. 22-34.

Misak, Ch. (2013). The American Pragmatist. Oxford: Oxford University Press. 
Nussbaum, M. C. (2010). Sin fines de lucro: por qué la democracia necesita de las Humanidades. (Trad. M.V. Rodil). Buenos Aires: Katz.

Rorty, R. (1996 [1982]). Consecuencias del pragmatismo. (Trad. J.M.E. Cloquell). Madrid: Tecnos.

Ryan, A. (1995). John Dewey and the High Tide of American Liberalism. Nueva York: W.W. Norton.

Shusterman, R. (2000). Pragmatist Aesthetics: Living Beauty, Rethinking Art. Lanham: Rowman and Littlefield.

----- (2002). Surface and Depth: Dialectics of Criticism and Culture. Ithaca y Londres: Cornell University Press.

Wexler, T. (2013). Collective Expressions: the Barnes Foundation and Philadelphia. Tesis de maestría. Bowling Green State University. Sin publicar.

Whitehead, A. N. (1948 [1925]). Science and the Modern World. Nueva York: Pelican Mentor Books. 